\title{
Investigating optical path and differential pathlength factor in reflectance photoplethysmography for the assessment of perfusion
}

Subhasri Chatterjee

Tomas Ysehak Abay

Justin Paul Phillips

Panayiotis A. Kyriacou 


\title{
Investigating optical path and differential pathlength factor in reflectance photoplethysmography for the assessment of perfusion
}

\author{
Subhasri Chatterjee, ${ }^{*}$ Tomas Ysehak Abay, Justin Paul Phillips, and Panayiotis A. Kyriacou \\ City, University of London, Research Centre for Biomedical Engineering, London, United Kingdom
}

\begin{abstract}
Photoplethysmography (PPG) is an optical noninvasive technique with the potential for assessing tissue perfusion. The relative time-change in the concentration of oxyhemoglobin and deoxyhemoglobin in the blood can be derived from DC part of the PPG signal. However, the absolute concentration cannot be determined due to the inadequate data on PPG optical paths. The optical path and differential pathlength factor (DPF) for PPG at red $(660 \mathrm{~nm})$ and infrared $(880 \mathrm{~nm})$ wavelengths were investigated using a heterogeneous Monte Carlo model of the human forearm. Using the simulated DPFs, the absolute time-change in concentrations were determined from PPG signals recorded from the same tissue site. Results were compared with three conditions of approximated DPFs. Results showed the variation of the optical-path and DPF with different wavelengths and source-detector separations. Approximations resulted in significant errors, for example, using NIRS DPF in PPG led to "cross talk" of -0.4297 and 0.060 and an error of $15.16 \%$ to $25.18 \%$. Results confirmed the feasibility of using the PPG (DC) for the assessment of tissue perfusion. The study also identified the inappropriateness of the assumption that DPF is independent of wavelength or source-detector separations and set the platform for further studies on investigating optical pathlengths and DPF in PPG. ๑ 2018 Society of Photo-Optical Instrumentation Engineers (SPIE) [DOI: 10.1117/1.JBO.23.7.075005]
\end{abstract}

Keywords: photoplethysmography; Beer-Lambert law; Monte Carlo model; optical path; near-infrared spectroscopy; oxy/deoxy hemoglobin.

Paper 170756RR received Nov. 21, 2017; accepted for publication Jun. 15, 2018; published online Jul. 11, 2018.

\section{Introduction}

Sufficient tissue perfusion is extremely vital for all metabolic processes in cells, including tissue repair and resistance to infectious organisms. Early detection of inadequate tissue perfusion is important for resuscitation and for the prevention of organ damage. To preserve adequate perfusion during circulatory failure, the blood supply is diverted from peripheral tissue to the main organs, such as brain, heart, and kidneys. ${ }^{1}$ In this scenario, monitoring cutaneous and subcutaneous perfusion could serve as an early biomarker for hypoperfusion in tissues and organs. The conventional assessment of tissue perfusion by global measurements, such as blood pressure, oxygen-derived variables, or blood lactate levels, have limitations regarding their accuracy and the invasiveness of the procedures. ${ }^{1-4}$

Photoplethysmography (PPG) is a noninvasive photometric technique used for the measurement of blood volume and is mostly known for its use in pulse oximeters for the measurement of arterial oxygen saturation, widely known as $\mathrm{SpO}_{2}$. Pulse oximeters had a large impact in modern clinical monitoring and nowadays are considered as a standard of care in anaesthesia and related clinical modalities. ${ }^{5}$ PPG has found its utility in a vast number of applications, such as neonatal care, hemorheology assessment, pulse transit time investigation, and measurements from body cavities and organs..$^{6-12}$

\footnotetext{
*Address all correspondence to: Subhasri Chatterjee, E-mail: subhasri
} .chatterjee.1@ city.ac.uk
Near-infrared spectroscopy (NIRS) is another noninvasive optical technique, which measures variations in local blood perfusion by estimating changes in oxy-hemoglobin, deoxyhemoglobin, and total hemoglobin. ${ }^{13}$ These physiological variables are determined using the Beer-Lambert law, correlating the attenuation of light $A$, through a medium with the concentration $C$ of the absorber (chromophore) present in the solution. With the corrections due to scattering, the modified BeerLambert law $^{14}$ used for the tissue medium can be expressed as follows:

$A_{\lambda}=\epsilon_{\lambda} \mathrm{Cl}_{\lambda}+G$

where $\lambda$ is the operating wavelength, $\epsilon_{\lambda}$ is the extinction coefficient (or molar absorptivity) of the chromophore, $l_{\lambda}$ is the optical pathlength through tissue, and $G$ is a geometry-dependent term, respectively. Due to multiple scattering, the physical pathlength traveled by the light through tissue is considerably higher than the source-detector (interoptode) separation employed in the measurement system. ${ }^{15}$ To consider this, a multiplicative term known as differential pathlength factor (DPF) was introduced to relate the actual light pathlength with the interoptode separation distance $d$ as follows:

$l_{\lambda}=\mathrm{DPF}_{\lambda} \cdot d$ 
Optical path and DPF have been estimated either experimentally or computationally through simulations of light diffusion in tissue, and they have been found to be dependent on the optical properties (mostly scattering) of the tissue, the light wavelength, and the geometry of the system. The DPF is always positive and $>1$, thus signifying an increase in the pathlength compared with the separation $d$. Empirical determinations of the DPF include measuring the time of flight by time-resolved spectroscopy (TRS) or relating the optical path to light intensities modulations in phase-resolved spectroscopy (PRS). ${ }^{13,16,17}$ Results from experimental studies showed that the DPF can be assumed relatively constant for a high source-detector separation $(d>2.5 \mathrm{~cm}) .^{18}$

Knowledge of the DPF is fundamental for the quantification of changes in oxy-hemoglobin and deoxyhemoglobin in tissues. Without the knowledge of the DPF, changes in oxy- and deoxyhemoglobin can only be expressed as relative to the optical pathlength (concentration $\times$ optical path). ${ }^{19}$ In continuous-wave NIRS instruments, fixed DPF values measured experimentally from tissues by TRS or PRS are conventionally used for the determination of 1 as in Eq. (2). As the estimation of changes in oxy-hemoglobin and deoxyhemoglobin in tissues requires the application of Eq. (1) at a minimum of two wavelengths (see later in Sec. 2), using a fixed DPF value assumes that the optical pathlength does not vary with the measuring wavelength (wavelength independence, i.e., $\mathrm{DPF}_{\lambda 1}=\mathrm{DPF}_{\lambda 2}$ ). Although offering a practical advantage, this assumption may differ from real situation theoretical models.

Recently, our research group has demonstrated the feasibility of using the photoplethysmographic signal for estimating changes in oxyhemoglobin $\left(\mathrm{HbO}_{2}\right)$, deoxyhemoglobin $(\mathrm{HHb})$, and total hemoglobin (tHb) as in NIRS. ${ }^{19,20}$ This can be achieved by splitting dual-wavelength PPG signals into AC and DC components and applying the modified Beer-Lambert law to the DC component. As the same PPG signals can also be used to estimate the conventional $\mathrm{SpO}_{2}$, this approach allows employing the same signals used in pulse oximetry for determining some hemodynamic parameters (i.e., oxy- and deoxy-hemoglobin) that can be useful in the assessment of perfusion changes. Although being relatively easy to apply and not requiring any additional instrumentation to the conventional pulse oximetry technology, this procedure is restricted by the unknown DPF. In the existing literature, ${ }^{18,21,22}$ the optical path has been evaluated for either longer source-detector separations $(>10 \mathrm{~mm})$ or for shorter source-detector separations $(<1 \mathrm{~mm})$. The available information is unlikely to match the source-detector separations, and also, the optical wavelengths, generally used in PPG sensors, thus limiting the quantification of the changes in oxy- and deoxy-hemoglobin from PPG as relative to the (unknown) optical pathlength.

Extensive work has previously been done to elucidate the distribution of light-tissue interactions, and assess the information on tissue oxygenation and perfusion, in the optical techniques, such as diffuse NIRS and laser Doppler flowmetry. ${ }^{15-18,22-28}$ Although some of these studies have determined the optical pathlength and DPF, others have assessed the oxy- and deoxyhemoglobin concentrations and the sources of errors in the measurement. However, to our knowledge, there has not been any previous attempt to investigate the optical pathlength and DPF for the quantification of oxy- and deoxyhemoglobin concentrations from PPG signals. Therefore, this present work aims at investigating the effects of pathlength assumptions in oxy- and deoxyhemoglobin measurements from dual-wavelength PPG signals by combining a computational model of light transport and experimental PPG measures.

A Monte Carlo (MC) model of light propagation was explored to compute the optical paths (and the DPF) through a heterogeneous tissue volume in a dual-wavelength reflectance PPG geometry. Moreover, the DPF at different wavelengths and as a function of various source-detector separation distances was analyzed, allowing the determination of the optical pathlengths for both large (NIRS) and small (PPG) interoptode separation distances. The DPF values computed by the model were then applied in the quantification of changes in hemoglobin concentrations from experimental PPG signals acquired in vivo from healthy volunteers. This also allowed to assess the errors introduced by DPF approximations in changes in oxy- and deoxyhemoglobin assessed by PPG.

\section{Theoretical Background}

The modified Beer-Lambert law, with the consideration that the main absorbers present in the tissue medium are oxyhemoglobin $\left(\mathrm{HbO}_{2}\right)$ and deoxyhemoglobin $(\mathrm{HHb})$ can be rewritten from Eq. (1) as follows:

$A_{\lambda}=\left(\epsilon_{\mathrm{HbO}_{2} \lambda} C_{\mathrm{HbO}_{2}}+\epsilon_{\mathrm{HHb} \lambda} C_{\mathrm{HHb}}\right) l_{\lambda}+G$,

where $\epsilon_{\mathrm{HbO}_{2} \lambda}$ and $\epsilon_{\mathrm{HHb} \lambda}$ are the extinction coefficients of oxyhemoglobin and deoxyhemoglobin, respectively, at wavelength $\lambda ; C_{\mathrm{HbO}_{2}}$ and $C_{\mathrm{HHb}}$ are the concentrations of oxyhemoglobin and deoxyhemoglobin, respectively. Considering $G$ to be constant over time, a differential approach of the modified BeerLambert law is applied, which permits the calculation of changes in concentrations relative to the baseline. Combining Eqs. (2) and (3), the time-changes in the light attenuation $(\Delta A)$ at the two operating wavelengths can be expressed as the functions of the changes in concentrations ${ }^{20}$ of oxyhemoglobin $\left(\Delta C_{\mathrm{HbO}_{2}}\right)$ and deoxyhemoglobin $\left(\Delta C_{\mathrm{HHb}}\right)$

$$
\begin{aligned}
& \Delta A_{\lambda 1}=\left(\epsilon_{\mathrm{HbO}_{2} \lambda 1} \cdot \Delta C_{\mathrm{HbO}_{2}}+\epsilon_{\mathrm{HHb} \lambda 1} \cdot \Delta C_{\mathrm{HHb}}\right) \cdot \mathrm{DPF}_{\lambda 1} \cdot d, \\
& \Delta A_{\lambda 2}=\left(\epsilon_{\mathrm{HbO}_{2} \lambda 2} \cdot \Delta C_{\mathrm{HbO}_{2}}+\epsilon_{\mathrm{HHb} \lambda 2} \cdot \Delta C_{\mathrm{HHb}}\right) \cdot \mathrm{DPF}_{\lambda 2} \cdot d .
\end{aligned}
$$

Equations (4) and (5) comprise a system of linear equations that can be solved as in Eqs. (6) and (7) for determining the two unknowns, $\Delta C_{\mathrm{HbO}_{2}}$ and $\Delta C_{\mathrm{HHb}}$

$$
\begin{aligned}
& \Delta C_{\mathrm{HbO}_{2}}=\frac{\frac{\Delta A_{\lambda 1} \epsilon_{\mathrm{HHb} \lambda 2}}{\mathrm{DPF}_{\lambda 1}}-\frac{\Delta A_{\lambda 2} \epsilon_{\mathrm{HHb} \lambda 1}}{\mathrm{DPF}_{\lambda 2}}}{d\left(\epsilon_{\mathrm{HbO}_{2 \lambda 1}} \epsilon_{\mathrm{HHb}_{\lambda 2}}-\epsilon_{\mathrm{HHb} \lambda 1} \epsilon_{\mathrm{HbO}_{2} \lambda 2}\right)}, \\
& \Delta C_{\mathrm{HHb}}=\frac{\frac{\Delta A_{\lambda 2} \epsilon_{\mathrm{HbO}_{2} \lambda 1}}{\mathrm{DPF}_{\lambda 2}}-\frac{\Delta A_{\lambda 1} \epsilon_{\mathrm{HbO}_{2} \lambda 2}}{\mathrm{DPF}_{\lambda 1}}}{d\left(\epsilon_{\mathrm{HbO}_{2 \lambda 1}} \epsilon_{\mathrm{HHb}_{\lambda 2}}-\epsilon_{\mathrm{HHb} \lambda 1} \epsilon_{\mathrm{HbO}_{2} \lambda 2}\right)} .
\end{aligned}
$$

Once $\Delta C_{\mathrm{HbO}_{2}}$ and $\Delta C_{\mathrm{HHb}}$ have been calculated, the time changes in total hemoglobin concentration $\Delta C_{\mathrm{tHb}}$ can also be derived as the sum of the two hemoglobins species 
$\Delta C_{\mathrm{tHb}}=\Delta C_{\mathrm{HbO}_{2}}+\Delta C_{\mathrm{HHb}}$

The impact of the DPF in the quantification of $\Delta C_{\mathrm{HbO}_{2}}$ and $\Delta C_{\mathrm{HHb}}$ can be seen in Eqs. (6) and (7), respectively, and it is clear that the inaccurate selection of DPF may cause systematic errors in the estimated concentration changes. ${ }^{23-25}$ The common assumption of wavelength independency in DPF (i.e., $\mathrm{DPF}_{\lambda 1}=\mathrm{DPF}_{\lambda 2}$ ) may result in "cross talk" between two hemoglobin species. Cross talk relates to the separability of the mixed optical signals into $\Delta C_{\mathrm{HbO}_{2}}$ and $\Delta C_{\mathrm{HHb}}$, and it is defined as the quantity of $\Delta C_{\mathrm{HbO}_{2}}$ that is reflected into $\Delta C_{\mathrm{HHb}}$ and viceversa. $^{24,25}$ The cross talk error (CT) due to inaccuracies in the optical pathlengths is expressed as

$\mathrm{CT}_{i \rightarrow j}=\frac{-\epsilon_{i \lambda 1} \epsilon_{i \lambda 2}}{\epsilon_{i \lambda 1} \epsilon_{j \lambda 2}-\epsilon_{j \lambda 1} \epsilon_{i \lambda 2}}\left[k_{\lambda 1}-k_{\lambda 2}\right]$,

where $\epsilon_{i}$ is the extinction coefficient of either oxyhemoglobin or deoxyhemoglobin, and $\epsilon_{j}$ is the extinction coefficient of the other species. $\mathrm{CT}_{i \rightarrow j}$ represents the cross talk from chromophore $i$ to chromophore $j$ and it is different in sign and magnitude from $\mathrm{CT}_{j \rightarrow i}$, which, in turn, is the cross talk from the chromophore $j$ to chromophore $i$. The term $k_{\lambda}$ is the relative pathlength factor $^{24,25}$ and mathematically represents the amount of error in the optical pathlengths at each wavelength. Here, we denote the simulated DPF using the MC model as $\mathrm{DPF}_{\mathrm{MC}}$ and the DPF approximated with the assumption of wavelength independency as $\mathrm{DPF}_{\text {approx }}$. Therefore, the relative pathlength factor can be expressed as follows:

$k_{\lambda}=\frac{\mathrm{DPF}_{\mathrm{MC}_{\lambda}}}{\mathrm{DPF}_{\text {approx }_{\lambda}}}$.

Cross talk provides the qualitative error information between the concentrations measured using real (simulated, in this case) and approximated DPF values. The quantitative (or absolute) errors between the concentration measured in two ways ( $E_{\mathrm{HHb}}$ and $E_{\mathrm{HbO}_{2}}$, respectively, for deoxy and oxyhemoglobin) can be derived directly, ${ }^{29}$ as shown in Eqs. (11) and (12)

$E_{\mathrm{HHb}}=\Delta C_{\mathrm{HHb}_{\mathrm{approx}}}-\Delta C_{\mathrm{HHb}_{\mathrm{MC}}}$,

$E_{\mathrm{HbO}_{2}}=\Delta C_{\mathrm{HbO}_{2} \text { approx }}-\Delta C_{\mathrm{HbO}_{2 \mathrm{MC}}}$.

The cross talk and errors quantify the differences between the concentrations evaluated using simulated and approximated DPF values. The simulation procedure will be described in the next sections, followed by the cross talk and error analysis of the recorded PPG signal.

\section{Methodology}

The block diagram of the simulation and experimental workflow used in this study is shown in Fig. 1. The first part of the work involved simulating an $\mathrm{MC}$ model of light propagation in the human forearm for the determination of optical paths and DPFs at different wavelengths. The second part consisted of investigating the effect of DPF approximations on the estimation of hemoglobin concentration changes from PPG signals. For this, in-vivo PPG measurements acquired at the forearm were used for the calculation of hemoglobin concentrations by applying three different DPF approximations (conditions A, B, and C). These hemoglobin concentrations were then compared

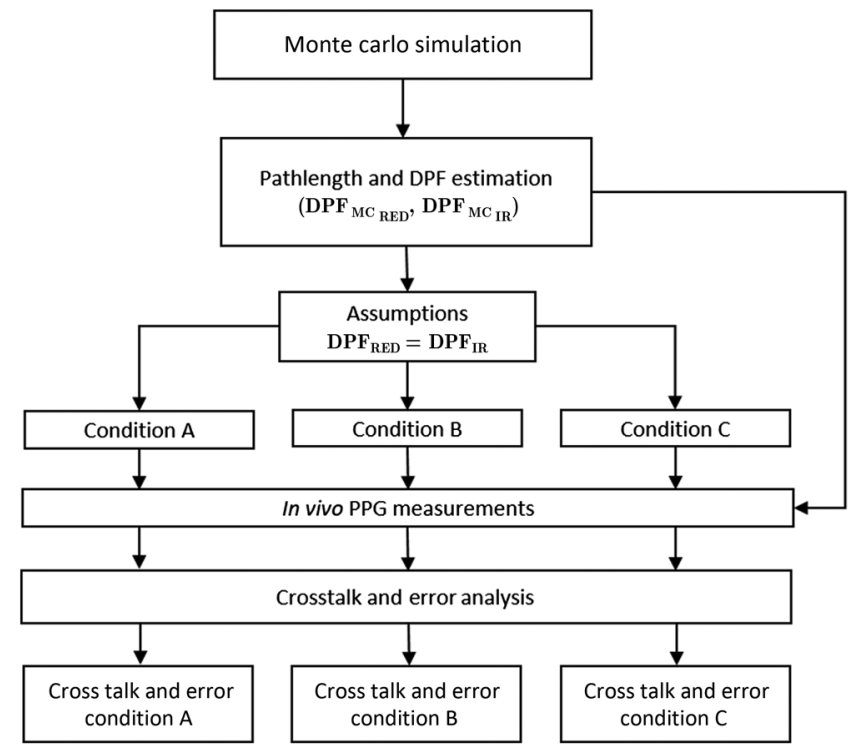

Fig. 1 Block diagram of the methodology.

with the same parameters calculated using the MC-simulated DPF values. Any inaccuracy introduced by the approximations was then quantified by estimating the cross talks and absolute errors in $\Delta C_{\mathrm{HBO}_{2}}$ and $\Delta C_{\mathrm{HHb}}$ measurements, respectively.

\subsection{Monte Carlo Simulation}

MC is a stochastic method used to solve complex tissue-opticsrelated problems. The basic recipe of an MC simulation has been explicitly described in the published literature ${ }^{29,30}$ and the method has been adopted as a primary tool by many researchers for the modeling of light-tissue interaction. ${ }^{31-35}$ In a typical MC model, virtual photon packets are generated and simulated through a number of random trajectories within a volume of tissue, whereas the optical path through the medium is determined by the wavelength-dependent optical properties of the tissue. In the current work, a heterogeneous MC model was prepared to simulate the optical properties of an experimental region of the body such as the human forearm. The model was explored for investigating optical paths and DPFs for a range of source-detector separations, from 2 to $40 \mathrm{~mm}$, with an interval of $1 \mathrm{~mm}$. The source-detector separation $d$ in our model was selected to investigate the optical path for a wide range of applications, including PPG (short separations) and NIRS (long separations).

\subsubsection{Tissue description}

A three-dimensional (3-D) slab geometry was chosen to represent the volume of the human forearm when interrogated by red $(660 \mathrm{~nm})$ and infrared $(880 \mathrm{~nm})$ light. To reconstruct a multilayer tissue model, the anatomical properties of the forearm were determined by surveying the published literature ${ }^{36-40}$ and analyzing histological images. The first six layers starting from the top represent the dermal sublayers of the skin (stratum corneum, epidermis, papillary dermis, upper blood net plexus, reticular dermis, and deep blood net plexus) and they were followed by the subdermal fat layer and the muscle layer. This order represented the first half of the tissue model and it was replicated in reverse order in the other half of the model. 


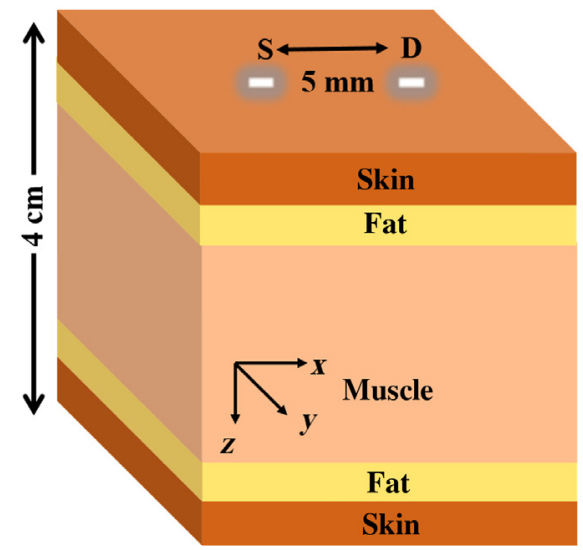

Fig. 2 Schematic of tissue layers of human forearm model presented in a 3-D Cartesian co-ordinate system. The model comprises of the layers skin, fat, muscle, and again fat and skin, respectively (skin sublayers are not shown separately). The depth of tissue is along the negative $z$-axis. Total tissue thickness is $4 \mathrm{~cm}$. In the reflectance PPG setting, the optical source $(S)$ and detector $(D)$ are placed at a distance of $5 \mathrm{~mm}$; however, the distance in the simulation was varied.

Table 1 Stratification order and properties of the tissue layers that were used for modeling the forearm. The thicknesses are presented with the volume distribution of blood and water in each layer. The simulated optical properties, such as absorption coefficient $\mu_{a}$, reduced scattering coefficient $\mu_{s}^{\prime}$, and anisotropy factor $g$ are listed for both 660 (red) and $880 \mathrm{~nm}$ (infrared).

\begin{tabular}{|c|c|c|c|c|c|c|}
\hline Tissue layer & $\begin{array}{l}\text { Thickness } \\
\text { (mm) }\end{array}$ & $C_{\text {blood }}$ & $C_{\text {water }}$ & $\begin{array}{l}\mu_{a}\left(\mathrm{~mm}^{-1}\right) \\
{\left[\frac{\text { red }}{\text { infrared }}\right]}\end{array}$ & $\begin{array}{l}\mu_{s}^{\prime}\left(\mathrm{mm}^{-1}\right) \\
{\left[\frac{\text { red }}{\text { infrared }}\right]}\end{array}$ & $g$ \\
\hline $\begin{array}{l}\text { 1. Stratum } \\
\text { corneum }\end{array}$ & 0.02 & 0 & 0.05 & $\frac{0.0497}{0.0222}$ & $\frac{2.562}{1.681}$ & 0.9 \\
\hline 2. Epidermis & 0.1 & 0 & 0.2 & $\frac{2.7316}{1.0592}$ & & \\
\hline $\begin{array}{l}\text { 3. Papillary } \\
\text { dermis }\end{array}$ & 0.2 & 0.04 & 0.5 & $\frac{0.0931}{0.0548}$ & & \\
\hline $\begin{array}{l}\text { 4. Upper blood } \\
\text { net plexus }\end{array}$ & 0.1 & 0.3 & 0.6 & $\frac{0.5128}{0.1666}$ & & \\
\hline $\begin{array}{l}\text { 5. Reticular } \\
\text { dermis }\end{array}$ & 1.23 & 0.04 & 0.7 & $\frac{0.0834}{0.0620}$ & & \\
\hline $\begin{array}{l}\text { 6. Deep blood } \\
\text { net plexus }\end{array}$ & 0.12 & 0.1 & 0.7 & $\frac{0.1814}{0.0869}$ & & \\
\hline $\begin{array}{l}\text { 7. Subdermal } \\
\text { fat }\end{array}$ & 3.23 & 0.05 & 0.7 & $\frac{0.0998}{0.0661}$ & $\frac{1.24}{1.09}$ & 0.8 \\
\hline 8. Muscle & 30 & 0.35 & 0.6 & $\frac{0.5944}{0.1874}$ & $\frac{0.8610}{0.635}$ & 0.9 \\
\hline
\end{tabular}

Overall, the multilayer tissue model comprised of 15 layers, with a total thickness of $4 \mathrm{~cm}$ and an infinite width (considering the spatial distribution of the photons to be much smaller than the width of the human forearm). Figure 2 shows the schematic of the tissue layers. Table 1 shows the order of the layers, their respective thicknesses, and optical properties. The effective absorption coefficient, $\mu_{a}(\lambda)$ of each tissue layer was calculated as the sum of the volumetric contributions of all absorbers present in the composition. ${ }^{35,41,42}$

The concentrations of blood $\left(C_{\text {blood }}\right)$ and water $\left(C_{\text {water }}\right)$ in each layer, used to simulate the overall perfusion and hydration of the tissue, ${ }^{34,35}$ are also shown in Table 1 . The arterial oxygen saturation of blood in all layers was considered $92 \%$, with an arteriovenous oxygen saturation difference of $10 \%$, an arteriovenous blood concentration ratio $1: 1$, and a hematocrit of $45 \%{ }^{43}$ A concentration of $10 \%$ melanin contributed in the total epidermal absorption. The absorption coefficients of water, melanin, and the two main absorbers in blood, i.e., oxy- and deoxyhemoglobin, were collected from the literature. ${ }^{44-48}$ The scattering coefficient $\mu_{s}(\lambda)$ and the anisotropy factor $g$ of all dermal layers, subdermal fat, and muscle tissue at 660 and $880 \mathrm{~nm}$ were adapted from the published data. ${ }^{49}$ Refractive index of tissue layers was taken to be 1.4 , and the external (air) refractive index was 1 , respectively.

\subsubsection{Method of execution}

In the MC model, the virtual photons were launched randomly into the tissue surface, following a Gaussian beam profile of $1-\mathrm{mm}$ radius. ${ }^{50}$ The optical path of the photon packet was simulated by tracing the photon's steps through the medium, determined by random sampling of the probability distribution $p(s)$ [Eq. (13)] for the photon's free pathlength $s$ between two consecutive interaction events

$p(s)=\mu_{s} \cdot \exp \left(-\mu_{s} . s\right)$.

The step size $s$ of a photon packet was calculated as follows:

$s=-\frac{\ln (\xi)}{\mu_{s}}$,

where $\xi$ is a computer-generated pseudorandom number lying between 0 and 1 . Once the step-size was determined, scattering was achieved by orienting the photon's direction through two randomly generated angles zenith $(\theta)$ and azimuth $(\phi)$, described, respectively, in Eqs. (15) and (16)

$\cos \theta=\frac{1}{2 g}\left[1+g^{2}-\left(\frac{1-g^{2}}{1-g+2 g \xi}\right)\right]$,

$\phi=2 \pi \xi$

Detailed calculations of the scattering angles and step sizes are described elsewhere. ${ }^{29,51}$ Each of the launched photon packets was introduced with an initial statistical weight $w=1$. After each step size, when the photon packet reached an interaction site, a fraction of its weight $\left(w \cdot \frac{\mu_{a}}{\mu_{a}+\mu_{s}}\right)$ was modeled to be absorbed within the tissue layer. The Russian roulette technique was used for the termination of the photon packets within the medium, weighing below a threshold weight ${ }^{29} w_{\text {the }}=10^{-4}$. If the photon packet was exiting the tissue surface, the fulfillment of the detection criteria was tested (i.e., if the photon had reached the circular area within 1-mm diameter from the detector), with the undetected photons leaving the tissue surface being discarded. The photon path was traced within tissue layers until it was detected, discarded, or terminated. The corrections 
due to reflection and refraction were considered each time the photon hits the boundary. The number of photons detected for the range of source-detector separations was $10^{10}$.

The total optical pathlength $(S)$ for each detected photon was determined as the sum of all the free pathlengths $\left(S=\sum s\right)$, whereas the mean optical path $l$ was calculated as the average of the pathlengths of all detected photons at each source-detector separation, i.e., $l=\bar{S}$. The photons were captured all over the range of source-detector separations, and then the averages of the optical paths were calculated for the photons reached in each detector, separated by a distance of $1 \mathrm{~mm} .{ }^{52}$ The DPF was calculated as the ratio of the calculated mean optical path $l$ and the source-detector separation $d\left(\mathrm{DPF}=\frac{l}{d}\right)$. At all distances, the DPF was calculated for both wavelengths, red $\left(\mathrm{DPF}_{\mathrm{MC}_{\mathrm{RED}}}\right)$ and infrared $\left(\mathrm{DPF}_{\mathrm{MC}_{\mathrm{IR}}}\right)$, respectively.

The MC model was designed in MATLAB ${ }^{\circledR}$ (Mathworks, Inc.). A 64-bit Operating System with an installed memory of $24 \mathrm{~GB}$ and an Intel ${ }^{\circledR}$ Xeon ${ }^{\circledR} \mathrm{CPU}(2.40 \mathrm{GHz}, 2$ processors $)$ was dedicated for the simulation. The code was written using "parallel programming" method utilizing an inbuilt MATLAB function in 16 parallel threads. It takes 5 to $8 \mathrm{~h}$ to detect $10^{10}$ photons within a source-detector separation within $10 \mathrm{~mm}$.

\subsection{In-Vivo Experimental Setting and Protocol}

The DPF values derived from the MC model were used for determining changes in the concentration of oxyhemoglobin and deoxyhemoglobin from in-vivo PPG measurements acquired from the forearm. The measurements were collected as a part of our previous studies ${ }^{20}$ and consisted of raw PPG signals $(\mathrm{AC}+\mathrm{DC})$ acquired at two wavelengths, red $(660 \mathrm{~nm})$ and infrared $(880 \mathrm{~nm})$, from a reflectance PPG sensor positioned on the volar side of the left forearm. The reflectance PPG sensor comprised of two red LEDs, two infrared LEDs, and a photodiode. The LEDs and photodiode were placed at a center-tocenter distance of $5 \mathrm{~mm}$.

The protocol consisted of a sequence of vascular occlusions, which aimed to induce changes in blood flow in the measurement area and they were performed by manually inflating a cuff positioned around the upper left arm (on the volar side of the brachioradialis muscle) of 21 healthy volunteers (13 males and 8 females having the mean age of 31.18 years with a standard deviation of 7.55). The protocol started with $5 \mathrm{~min}$ of baseline measurements, followed by two minutes of venous occlusion $(60-\mathrm{mmHg}$ occlusion pressure), 2 min of recovery (no pressures), and two final minutes of total vascular occlusion (occlusion pressure $20-\mathrm{mmHg}$ over the systolic pressure).

The postprocessing of the signals was performed on MATLAB. The DC components were obtained with a low-pass zero-phase digital filter (cutoff frequency: $0.1 \mathrm{~Hz}$ ). A more detailed description of the protocol and the reflectance PPG sensor can be found in a previous publication. ${ }^{19}$

\subsection{Data Analysis}

The changes in concentration in oxy-, deoxy-, and total hemoglobin were calculated by applying the modified Beer-Lambert law to the red and infrared PPG signals acquired from the forearm, following Eqs. (6)-(8). The extinction coefficients for the estimation of $\mathrm{HbO}_{2}$ and $\mathrm{HHb}$ were obtained from published data on human blood spectra. ${ }^{53}$ The hemoglobin concentrations were calculated using different DPFs to investigate the effects of
DPF approximations on $\Delta C_{\mathrm{HbO}_{2}}$ and $\Delta C_{\mathrm{HHb}}$ estimated from PPG measurements.

In the first instance, the DPF values for red and infrared obtained through the $\mathrm{MC}$ simulation $\left(\mathrm{DPF}_{\mathrm{MC}_{\mathrm{RED}}}\right.$ and $\left.\mathrm{DPF}_{\mathrm{MC}_{\mathrm{IR}}}\right)$ were utilized for calculating $\Delta C_{\mathrm{HbO}_{2}}$ and $\Delta C_{\mathrm{HHb}}$ from the PPGs acquired from the forearm. This situation could be considered as "real" in this case as the DPFs were obtained from the simulation of a realistic model and no assumption or approximation was made regarding the optical pathlength (i.e., $\left.\mathrm{DPF}_{\mathrm{RED}} \neq \mathrm{DPF}_{\mathrm{IR}}\right)$.

After this, the PPG signals were also used to estimate $\Delta C_{\mathrm{HbO}_{2}}$ and $\Delta C_{\mathrm{HHb}}$ using DPF values under three different sets of approximations. In each condition, the DPF was assumed equal at both wavelengths (wavelength independency). For the first condition (condition A), the DPF for both wavelengths was set equal to the value simulated for the red wavelength $\left(\mathrm{DPF}_{A_{\mathrm{RED}}}=\mathrm{DPF}_{\mathrm{MC}_{\mathrm{RED}}}\right.$ and $\left.\mathrm{DPF}_{A_{\mathrm{IR}}}=\mathrm{DPF}_{\mathrm{MC}_{\mathrm{RED}}}\right)$. Similarly, in the second condition (condition $\mathrm{B}$ ), the DPF was set equal to the value obtained for the infrared wavelength from the MC simulation $\left(\mathrm{DPF}_{B_{\mathrm{RED}}}=\mathrm{DPF}_{\mathrm{MC}_{\mathrm{IR}}}\right.$ and $\left.\mathrm{DPF}_{B_{\mathrm{IR}}}=\mathrm{DPF}_{\mathrm{MC}_{\mathrm{IR}}}\right)$. Finally, in the third condition (condition C), the DPF was again assumed equal for both wavelengths but selecting an experimentally measured DPF for NIRS measurements from the existing NIRS literature, ${ }^{21}$ where the parameter was measured from the adult forearm by the time of flight method $(\lambda=761 \mathrm{~nm})$, resulting in a value of 3.59 for long separation distances $(d>2.5 \mathrm{~cm})$. The DPFs in condition $\mathrm{C}$ were then set to values equal to 3.59 for both wavelengths $\left(\mathrm{DPF}_{C_{\mathrm{RED}}}=3.59\right.$ and $\mathrm{DPF}_{C_{\mathrm{IR}}}=3.59$ ).

For each of the conditions where the DPFs were approximated (i.e., A, B, and C), the cross talk and absolute errors were assessed against the results obtained with the MCsimulated DPFs determined by the $\left(\mathrm{DPF}_{\mathrm{MC}_{\mathrm{RED}}}\right.$ and $\left.\mathrm{DPF}_{\mathrm{MC}_{\mathrm{IR}}}\right)$. The cross talk between hemoglobin species was determined using Eq. (9), with the relative pathlength factor $k_{\lambda}$ being set as the ratio between the DPF obtained at each wavelength from the MC simulation and the DPFs assumed at each of the three conditions above, for example, $k_{A_{\text {red }}}=\frac{\mathrm{DPF}_{\mathrm{MC}_{\mathrm{RED}}}}{\mathrm{DPF}_{A_{\mathrm{RED}}}}$ and so on. The inaccuracies in the hemoglobin concentrations in the three conditions were assessed by determining the absolute error in the measurements. This was obtained for each hemoglobin species by computing the maximum difference between the concentrations estimated using simulated DPFs $\left(\mathrm{DPF}_{\mathrm{MC}}\right)$ and approximated DPFs $\left(\mathrm{DPF}_{\text {approx }}\right)$ as shown in Eqs. (11) and (12). The error was then normalized to the full measurement range and expressed as a percentage error. The values across the population were presented as median and interquartile ranges (IQR).

\section{Results}

\subsection{Simulation of Scattering Distribution}

Figures 3(a) and 3(b) show the density plots of the simulated photon migration in the forearm at both red and infrared (only detected photon distributions are shown). The plots show the colourmap of the scattering events $N$ for red and infrared reflectance PPG measurements, acquired with an interoptode separation distance of $5 \mathrm{~mm}$. As the number of scattering events represents the accumulation of photons, it is clearly visible that the maximum number of photons gathers within the top few layers in the proximity of the light source and detector. 


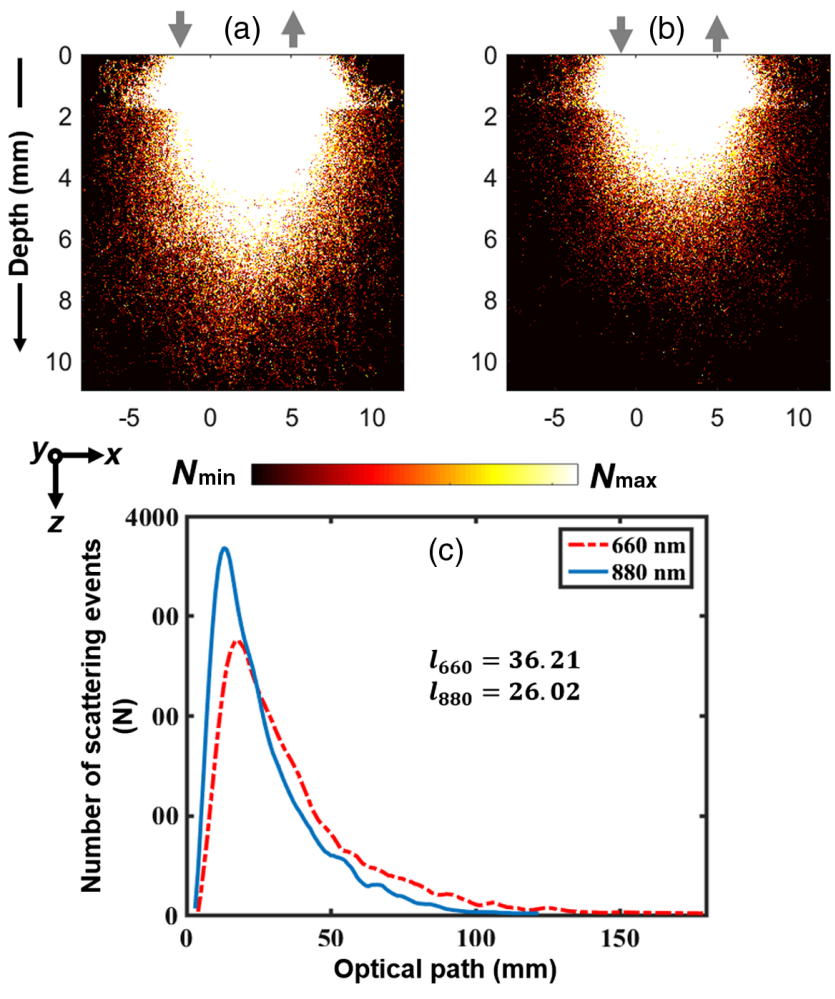

Fig. 3 Simulated distributions of scattering events $(N)$ through human forearm in PPG measurements at (a) $660 \mathrm{~nm}$ and (b) $880 \mathrm{~nm}$. The images are 2-D projections of the 3-D model, with a source-detector separation distance fixed at $5 \mathrm{~mm}$. Only the photons that reach the detector from the source are shown. The images are scaled equally. Colormap represents distribution between maximum $\left(N_{\max }\right)$ and minimum $\left(N_{\min }\right)$ values of number of scatter. The number of distribution of photon scatter events with the optical path $(S)$ is shown in (c), with red dashed and blue solid lines representing 660- and 880-nm wavelengths, respectively.

The photon scattering distribution clearly decreases with an increase in the depth. It is noticeable that the majority of the scattering events for both wavelengths take place within the dermal skin layers, extending up to a depth of $1.77 \mathrm{~mm}$. Almost no photons travel any deeper than the fat tissue layer (i.e., 5-mm depth from the surface). Figure 3(c) shows the distribution of the scattering events as a function of the optical pathlength $S$. As visually shown in Fig. 3(c), the number of scattering events is concentrated at short optical pathlengths (i.e., short penetration). With increasing optical pathlength, the photon packets diminish at both wavelengths but with a faster decrease at $880 \mathrm{~nm}$ compared with $660 \mathrm{~nm}$. Overall, the total optical path for red and infrared extended, respectively, to 80 and $122 \mathrm{~mm}$. The mean optical path $l=\bar{S}$ calculated for red and infrared wavelengths are 36.21 and $26.02 \mathrm{~mm}$, respectively.

In Fig. 4, the scattering distributions for higher sourcedetector separations, which are beyond usual PPG specifications, and fall normally within the NIRS source-detector separation range, are shown. Simulations were performed for the separations 15,25 , and $35 \mathrm{~mm}$. It is found that the penetration depth increases slowly with increasing source-detector separation. Also, in each case, the red penetration depth is higher compared with infrared. However, even for a high source-detector separation, the photons do not penetrate beyond the muscle tissue layer in the forearm.
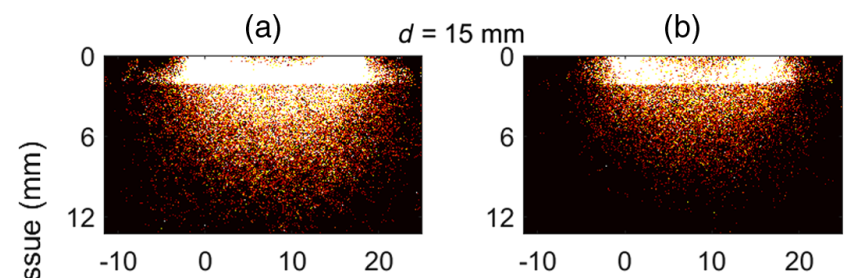

(c)

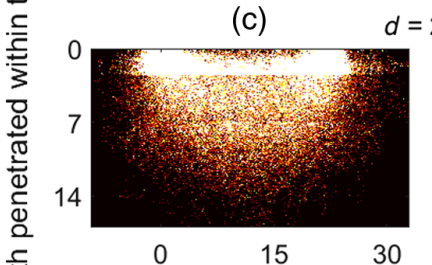

(d)
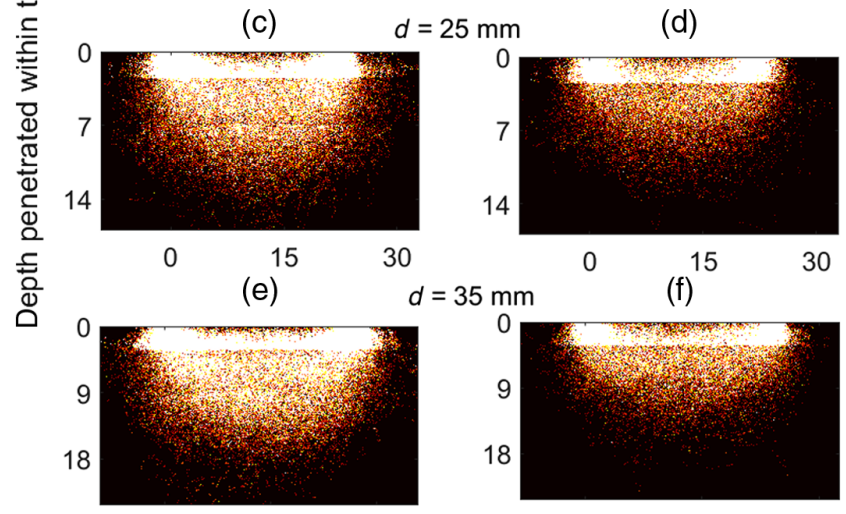

$0 \quad 20$

40 $d=35 \mathrm{~mm}$

(f)

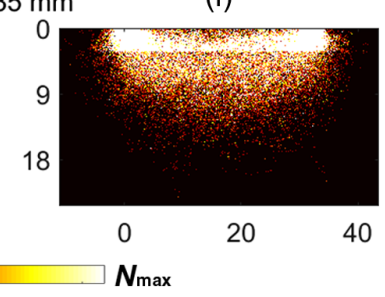

Fig. 4 Simulated distributions of scattering events $(N)$ through human forearm at 660 and $880 \mathrm{~nm}$ for larger source-detector separations: (a) and (b) $d=15 \mathrm{~mm}$, (c) and (d) $d=25 \mathrm{~mm}$, and (e) and (f) $d=35 \mathrm{~mm}$, respectively. The images are 2-D projections of the 3-D model. Only the photons that reach the detector from the source are shown. Colormap represents the distribution between maximum $\left(N_{\max }\right)$ and minimum $\left(N_{\min }\right)$ values of number of scatter.

(a)

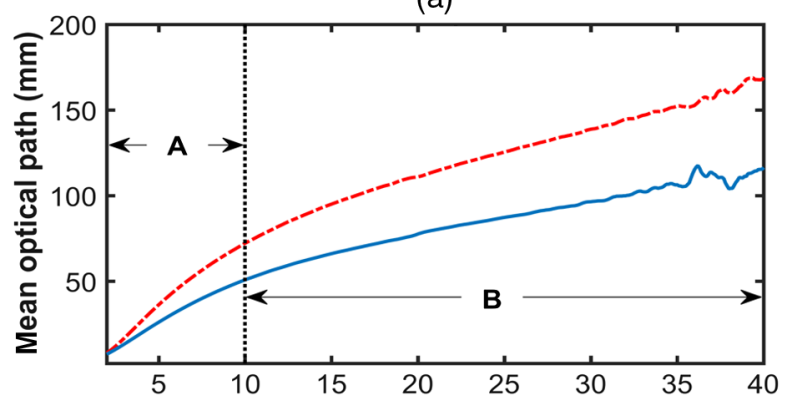

(b)

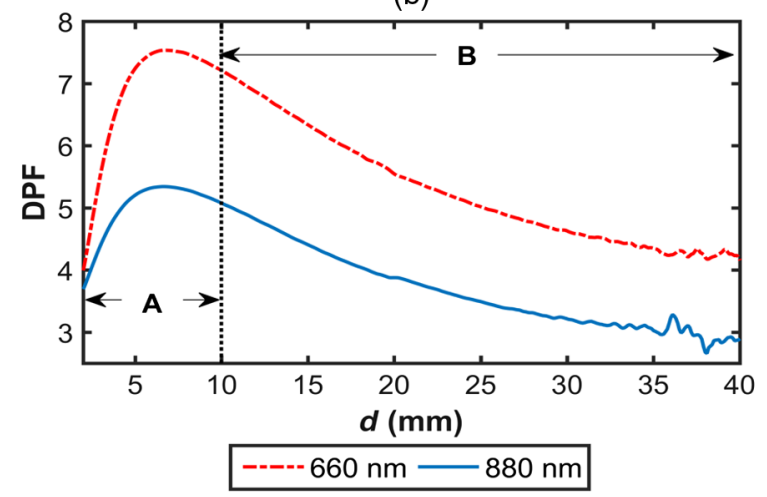

Fig. 5 (a) Mean optical path / and (b) DPF simulated through the human forearm at wavelengths 660 and $880 \mathrm{~nm}$, as functions of source-detector separation $d$. The region of shorter $d$ is marked as (A) and the region at longer $d$ is marked at (B), representing, respectively, the PPG and NIRS regions of measurements. Red dashed lines represent the results from $660 \mathrm{~nm}$, whereas blue solid lines represent the $800 \mathrm{~nm}$. 
Table 2 DPF at both red and infrared wavelengths for different source-detector separation distances $d$. The values are derived from the MC simulations of light transport through human forearm. The mean optical pathlength $I$ can be calculated as $I=d \times$ DPF.

\begin{tabular}{lcc}
\multirow{2}{*}{$\begin{array}{l}\text { Source-detector } \\
\text { separation } d(\mathrm{~mm})\end{array}$} & \multicolumn{2}{c}{ DPF } \\
\cline { 2 - 3 } 2 & $660 \mathrm{~nm}$ & $880 \mathrm{~nm}$ \\
\hline 3 & 3.998 & 3.70 \\
4 & 5.542 & 4.398 \\
5 & 6.649 & 4.915 \\
6 & 7.242 & 5.203 \\
7 & 7.487 & 5.323 \\
10 & 7.532 & 5.337 \\
20 & 7.213 & 5.077 \\
30 & 5.547 & 3.876 \\
40 & 4.628 & 3.215 \\
\hline
\end{tabular}

\subsection{Optical Pathlength and Differential Pathlength Factor}

Figures 5(a) and 5(b) show the simulated distributions of the mean optical path $(l)$ and DPF for the human forearm at red and infrared. The plots show the results of $l$ and DPF for a wide range of source-detector separation distances $(d)$, ranging from 2 to $40 \mathrm{~mm}$. As expected, the mean optical path $l$ traveled by the photons increased with the separation distance $d$, indicating a deeper light penetration with increasing $d$. The differences in $l$ and DPF at the two different wavelengths are clearly visible, with the optical path at $660 \mathrm{~nm}$ being $>880 \mathrm{~nm}$, thus resulting in a greater DPF at the red wavelength. The plots in Figs. 5(a) and 5(b) were divided into two application regions, depending on the source-detector separation distance $d$. The region A, at shorter $d$, represents the typical application region of PPG measurements, whereas the region $\mathrm{B}$ indicates the applications with medium-large separation distances. The upper end of region B mainly represents NIRS applications, consisting in very large source-detector separation distances $(d>2.5 \mathrm{~cm})$. Interestingly, the increase of $l$ with $d$ in the PPG region (A) was much sharper compared with the NIRS region (B), resulting in a steep increase in DPF for lower $d$.

For DPFs $>30 \mathrm{~mm}$ (region B), the DPFs at both red and infrared wavelengths tended to an asymptote (constant DPF), with a concomitant decrease in the difference between red and infrared DPFs. Table 2 shows some DPF values at 660 and $880 \mathrm{~nm}$ for different separation distances $d$ commonly employed in PPG and NIRS measurements.

\subsection{Experimental In-Vivo Results}

Figure 6 shows the changes in the concentration of $\mathrm{HbO}_{2}$ and $\mathrm{HHb}$ from a subject, estimated from PPG signals in the three conditions (described in Sec. 3.3). The trends in the changes of $\Delta C_{\mathrm{HbO}_{2}}$ and $\Delta C_{\mathrm{HHb}}$ indicate the variations in blood flow, either of venous or arterial nature, caused by the vascular occlusions. For comparison, the $\Delta C_{\mathrm{HbO}_{2}}$ and $\Delta C_{\mathrm{HHb}}$ obtained with the simulated DPFs are plotted as well in the same figure, showing how the approximations in the DPFs introduced some discrepancies in the estimation of $\Delta C_{\mathrm{HbO}_{2}}$ and $\Delta C_{\mathrm{HHb}}$. The differences in the estimation of $\Delta C_{\mathrm{HbO}_{2}}$ and $\Delta C_{\mathrm{HHb}}$ were assessed by calculating the cross talk between the two hemoglobin species. Table 3 shows the values of cross talk calculated in all the three different conditions investigated, and it shows how the approximations in all three conditions resulted in a consistent cross talk between the hemoglobin species. The highest degree of cross talk for both hemoglobin species was observed in condition $\mathrm{C}$, where a DPF for NIRS measurements was used for estimating $\Delta C_{\mathrm{HbO}_{2}}$ and $\Delta C_{\mathrm{HHb}}$ from PPG signals.

When compared with the results obtained with the DPFs from the MC simulations, the use of approximated DPFs for the estimation $\Delta C_{\mathrm{HbO}_{2}}$ and $\Delta C_{\mathrm{HHb}}$ during occlusions also resulted in a consistent error in the quantification of the magnitude of change. Figure 7 shows the boxplots of the normalized percentage errors in $\Delta C_{\mathrm{HbO}_{2}}$ and $\Delta C_{\mathrm{HHb}}$ in the three different conditions. The normalized maximum errors in $\Delta C_{\mathrm{HbO}_{2}}$ were $26.04(\mathrm{IQR}=21.76$ to 27.32$) \%, 9.90 \quad(\mathrm{IQR}=7.25$ to $12.28) \%, 19.53(\mathrm{IQR}=15.16$ to 25.18$) \%$, respectively, for conditions $\mathrm{A}, \mathrm{B}$, and $\mathrm{C}$. The normalized errors for $\Delta C_{\mathrm{HHb}}$ were 3.17 $(\mathrm{IQR}=2.18$ to 4.85$) \%, 13.44$ (IQR $=13.00$ to 15.83$) \%$, and 33.43 (IQR $=32.18$ to 39.39$) \%$ for conditions $\mathrm{A}, \mathrm{B}$, and $\mathrm{C}$, respectively.

\section{Discussion}

The knowledge of the pathlength traveled by the light in tissue is of fundamental importance for the determination of perfusion parameters such as the concentrations of oxy- and deoxyhemoglobin. However, the incorrect use of optical pathlengths or DPFs causes quantification errors in the measurements or cross talk between the hemoglobin species. Currently, such knowledge can be acquired by MC simulations of photons distribution or with experimental measures. This paper focuses on investigating the optical pathlength and DPF in reflectance PPG for different source-detector separation distances.

The tissue model described in this work considers baseline parameters and properties, with blood volume, water concentration, and melanin concentration being extracted from the literature. The blood oxygen saturation was consistent with the arterial blood oxygen saturation observed from the experimental forearm measurements. The arteriovenous difference in blood volume and oxygen saturation was selected based on previously published literature, representing the ideal scenario of a healthy subject, which, however, may differ for cases such as critically ill patients.

The first part of this paper focuses on the MC simulation of photons distribution to determine the optical path of PPG measurements at the forearm. In the results from the MC simulation, the optical path at red wavelength was higher than the optical path at infrared for all the source-detector separations investigated. This finding is consistent with our previously published results on a monolayer skin tissue model, where the red optical path was always greater than infrared for a range of sourcedetector separations and for different blood volumes and oxygen saturations. ${ }^{52,54}$

From the simulation results, both red and infrared light penetrated a certain distance within the tissue, with maximum 

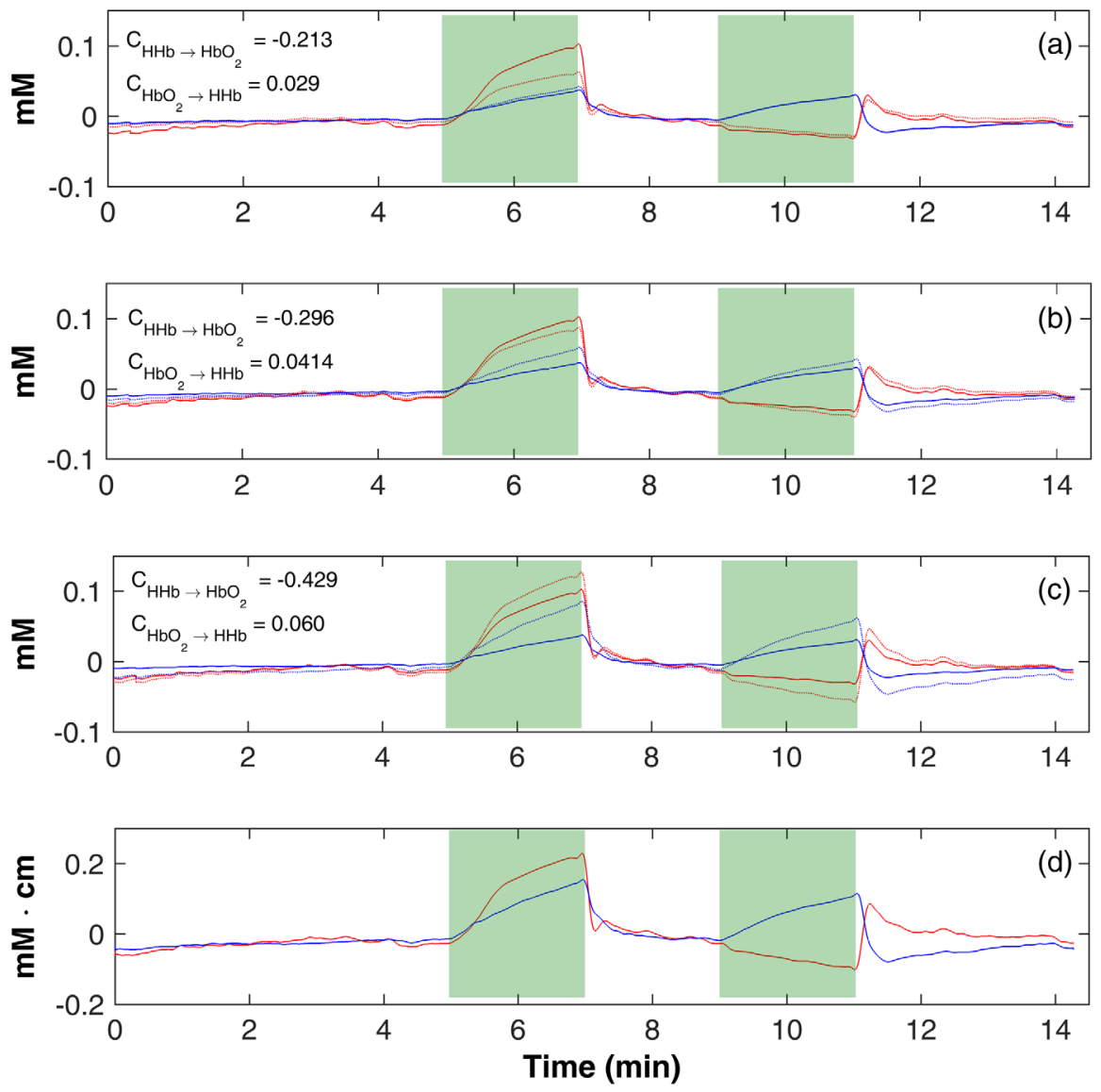

Fig. 6 Changes in concentrations of oxyhemoglobin $\mathrm{HbO}_{2}$ (red traces) and deoxyhemoglobin $\mathrm{HHb}$ (blue traces) estimated from a subject during the protocol in the three conditions investigated. The solid lines represent the changes estimated with the simulated DPFs, whereas the dotted lines represent the same estimated with the approximated DPFs. (a) $\Delta C_{\mathrm{HbO}_{2}}$ and $\Delta C_{\mathrm{HHb}}$ in condition A, (b) $\Delta C_{\mathrm{HbO}_{2}}$ and $\Delta C_{\mathrm{HHb}}$ in condition $\mathrm{B}$, and (c) $\Delta C_{\mathrm{HbO}_{2}}$ and $\Delta C_{\mathrm{HHb}}$ in condition $\mathrm{C}$. The bottom trace shows the $\Delta C_{\mathrm{HbO}_{2}}$ and $\Delta C_{\mathrm{HHb}}$ estimated without DPF (concentration $\times$ optical pathlength). The shaded green areas indicate the duration of the venous occlusion (minutes 5 to 7 ) and total occlusion (minutes 9 to 11). For improved clarity, plots $A, B$, and $C$ have been plotted with equal scales, whereas the last instance [(d) no DPF] has been plotted with a different scale in mM.cm.

Table 3 Cross talk between hemoglobin species calculated for the three conditions investigated. $\mathrm{CT}_{\mathrm{HHb} \rightarrow \mathrm{Hbo}_{2}}$ is the cross talk of deoxyhemoglobin into oxyhemoglobin, whereas $\mathrm{CT}_{\mathrm{Hbo}_{2} \rightarrow \mathrm{HHb}}$ represents the cross talk of oxy-hemoglobin into deoxyhemoglobin.

\begin{tabular}{lcc} 
& \multicolumn{2}{c}{ Cross talk } \\
\cline { 2 - 3 } & $\mathrm{CT}_{\mathrm{HHb} \rightarrow \mathrm{Hbo}_{2}}$ & $\mathrm{CT}_{\mathrm{Hbo}_{2} \rightarrow \mathrm{HHb}}$ \\
\hline Condition A & -0.213 & 0.0297 \\
Condition B & -0.2965 & 0.0414 \\
Condition C & -0.4297 & 0.060 \\
\hline
\end{tabular}

scattering in the first few layers of the skin and a reduction of scattering in the fat and muscle layers. The optical paths were mainly limited within the cutaneous and subcutaneous tissue region, which indicates that PPG would only provide shallow tissue perfusion and not deep tissue perfusion as NIRS. In the two application regions investigated (i.e., short separation $d$ for PPG and long separations for NIRS), the optical pathlength and DPF showed very different characteristics. This dynamic
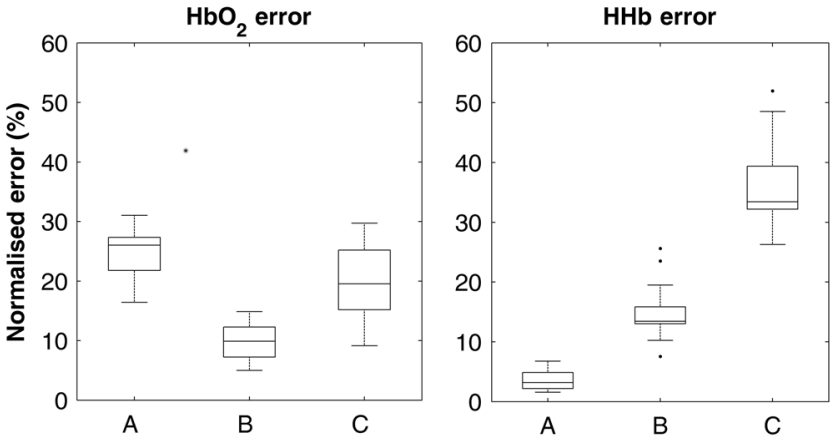

Fig. 7 Boxplots of the normalised percentage errors of $\Delta C_{\mathrm{HbO}_{2}}$ and $\Delta C_{\mathrm{HHb}}$ in the three conditions $(\mathrm{A}, \mathrm{B}$, and $\mathrm{C})$ investigated. The values represent the maximum absolute errors observed in $\Delta C_{\mathrm{HbO}_{2}}$ and $\Delta C_{\mathrm{HHb}}$ from the $\mathrm{MC}$ simulation condition, normalized to the full measurement ranges.

behavior of the DPFs at short separation distances should be taken into consideration in the use of an appropriate DPF for the quantification of hemoglobin concentration changes from PPG signals. Overall, the DPF for the red wavelength was always greater than infrared, thus highlighting a consistent 
wavelength-dependency of the DPF for short separation distances.

Optical path depends on the scattering properties of the medium. The scattering coefficients of all tissue layers possess higher values in red wavelength compared with the infrared wavelength (according to Table 1), producing higher red optical paths for all source-detector separation. Consequently, DPF at $660 \mathrm{~nm}$ is consistently $>880 \mathrm{~nm}$. The decline in the measured DPF values with increasing wavelength was observed in the literature by Duncan et al. ${ }^{18}$ in their experimental measurements of DPF on the forearm. The behavior of DPF for varying $d$ that was observed in this study agrees with experimental measurements of DPF previously reported by van der Zee et al., ${ }^{21}$ in which the DPF was measured by TRS (time of flight) at interoptode separation distances ranging from 10 to $60 \mathrm{~mm}$. In their measurements on the forearm, the authors reported a gradual decrease in DPF with increasing interoptode distance, similarly observed in our MC simulation. Also, van der Zee et al. ${ }^{21}$ observed that the measured DPFs were relatively constant for separation distances exceeding $25 \mathrm{~mm}$. However, the DPF for separation $d<10 \mathrm{~mm}$ (i.e., PPG applications) was never measured, which thus motivates us in investigating this parameter for dual-wavelength PPG through MC simulations.

This paper also wanted to investigate the effects of DPFs approximations on the estimation of hemoglobin concentration changes from PPG signals. Approximations on the DPFs from the MC simulation were performed and the hemoglobin concentrations were estimated from in vivo PPG signals with these approximated values. The results showed that assumptions or approximations in DPF could have a major impact in the quantification of $\Delta C_{\mathrm{HbO} 2}$ and $\Delta C_{\mathrm{HHb}}$ from PPG signals. Using inadequate DPFs appeared to cause cross talk between hemoglobin species, decreasing the capability to separate $\Delta C_{\mathrm{HbO} 2}$ and $\Delta C_{\mathrm{HHb}}$ and vice versa. This can also be reflected in the significant differences in the magnitude of the changes observed, which caused errors in the measurements. Using a DPF for NIRS measurements (i.e., condition $\mathrm{C}$ ) caused the largest cross talk and errors for both $\Delta C_{\mathrm{HbO} 2}$ and $\Delta C_{\mathrm{HHb}}$. In addition to assuming wavelength independency of DPF, this condition implied the use of a DPF that is intended for longer separation distances at a different wavelength (i.e., $761 \mathrm{~nm}$ ). This suggests that for the quantification of hemoglobin concentrations from PPG, the use of DPFs from the NIRS literature should be avoided and could cause significant measurement errors.

Even though approximations on the DPFs caused cross talk between hemoglobin species and magnitude errors, this did not seem to affect the ability of the hemoglobin concentration changes in detecting variations in perfusion during the vascular occlusions. The trends in the changes in hemoglobin concentrations from PPG showed consistent changes in venous and total occlusions, thus maintaining the ability of the PPG to detect changes in perfusion. However, it is not known at which level this will impact the ability of PPG. Differently from NIRS, where the DPF can be assumed constant for long separation distances, approximating the DPF in PPG measurements could cause quantification errors. Therefore, in the absence of the correct DPFs (either simulated or experimentally measured), researchers should express the hemoglobin concentrations from PPG as relative to the optical pathlength, i.e., (absolute change in concentration) $\times$ (optical pathlength) in the units of mM.cm. ${ }^{19,20}$ This more careful approach will avoid quantification errors and misleading results.
The model used in this work has not included the optical properties of the bone layer. Due to the high scattering and reflecting optical characteristics of bone, especially in the present source-detector geometrical setting, the photons scattered within the bone are very unlikely to survive until they reach the detector. ${ }^{37,41}$ Therefore, the presence of bone layers in the forearm (radius and ulna, which are two cylindrical bones, deep below the skin and surrounded by muscle tissue $^{36}$ ) was not considered in the model. The "Russian roulette" paradigm in the model was used to facilitate an unbiased termination, maintaining the energy conservation principle. ${ }^{30}$ Adding the additional weight each time to the photon might lead to an increase of photon pathlength in the medium, the physical interpretation of which is complicated. ${ }^{55}$ However, this did not induce any error in the results from the present model having the sensor-tissue geometry used in PPG, as the photon packets were detected with their weights higher than the threshold, thus did not undergo the Russian roulette.

\section{Conclusion}

A multilayer MC model of the human forearm was rigorously investigated to find the optical path and DPF in PPG at red $(660 \mathrm{~nm})$ and infrared $(880 \mathrm{~nm})$ wavelengths in an effort to derive the time changes in concentrations of oxyhemoglobin and deoxyhemoglobin with the modified Beer-lambert law. The optical paths and DPFs have been found to behave differently, depending on the interoptode separations and wavelengths. To study the influence of the DPF values on the concentration measurements from PPG, recorded PPG signals from the human forearm were analyzed using the simulated and approximated DPFs. The study showed cross talk and absolute errors in the approximated values, pointing toward the proper evaluation of DPF's for PPG instead of crude assumptions. The present study shows for the first time that a combination of simulation and experimental work can contribute in new knowledge and capabilities of the PPG beyond its conventional use in oxygen saturation measurement and toward the assessment of tissue perfusion.

\section{Disclosures}

The authors have no relevant financial interests in this article and no potential conflicts of interest to disclose.

\section{References}

1. A. Lima and J. Bakker, "Noninvasive monitoring of peripheral perfusion," Intensive Care Med. 31(10), 1316-1326 (2005).

2. J. Bakker et al., "Blood lactate levels are superior to oxygen-derived variables in predicting outcome in human septic shock," Chest 99(4), 956-962 (1991).

3. D. De Backer et al., "Microvascular blood flow is altered in patients with sepsis," Am. J. Respir. Crit. Care Med. 166(1), 98-104 (2002).

4. M. Y. Rady and E. P. Rivers, "The responses of blood pressure, heart rate, shock index, central venous oxygen saturation and lactate to resuscitation in the emergency department," Crit. Care Med. 23, A138 (1995).

5. P. A. Kyriacou, "Pulse oximetry in the oesophagus," Physiol. Meas. 27(1), R1-R35 (2006).

6. J. M. May, P. A. Kyriacou, and A. J. Petros, "A novel fontanelle probe for sensing oxygen saturation in the neonate," Biomed. Phys. Eng. Express 3(6), 015023 (2017).

7. J. Allen, "Photoplethysmography and its application in clinical physiological measurement," Physiol. Meas. 28(3), R1-R39 (2007).

8. N. Haneen and P. A. Kyriacou, "Photoplethysmography for the Assessment of Haemorheology," Sci. Rep. 7(1), 1406 (2017). 
9. K. Budidha and P. A. Kyriacou, "Investigation of pulse transit times utilizing multisite reflectance photoplethysmography under conditions of artificially induced peripheral vasoconstriction," in Conf. Proc. IEEE Engineering in Medicine and Biology Society (2014).

10. M. Hickey et al., "Investigation of photoplethysmographic signals and blood oxygen saturation values obtained from human splanchnic organs using a fiber optic sensor," J. Clin. Monit. Comput. 25(4), 245-255 (2011).

11. M. Hickey et al., "An in vivo investigation of photoplethysmographic signals and preliminary pulse oximetry estimation from the bowel using a new fiberoptic sensor," Anesth. Analg. 112(5), 1104-1109 (2011).

12. K. Budidha and P. A. Kyriacou, "In vivo investigation of ear canal pulse oximetry during hypothermia," J. Clin. Monit. Comput. 32(1), 97-107 (2018).

13. P. Rolfe, "In vivo near-infrared spectroscopy," Annu. Rev. Biomed. Eng. 2(1), 715-754 (2000).

14. L. Kocsis, P. Herman, and A. Eke, "The modified Beer-Lambert law revisited," Phys. Med. Biol. 51(5), N91-N98 (2006).

15. D. T. Delpy et al., "Estimation of optical pathlength through tissue from direct time of flight measurement," Phys. Med. Biol. 33(12), 1433-1442 (1988).

16. D. T. Delpy and M. Cope, "Quantification in tissue near-infrared spectroscopy," Philos. Trans. R. Soc. B Biol. Sci. 352(1354), 649-659 (1997).

17. M. Ferrari, L. Mottola, and V. Quaresima, "Principles, techniques, and limitations of near infrared spectroscopy," Can. J. Appl. Physiol. 29(4), 463-487 (2004)

18. A. Duncan et al., "Optical pathlength measurements on adult head, calf and forearm and the head of the newborn infant using phase resolved optical spectroscopy," Phys. Med. Biol. 40(2), 295304 (1995).

19. T. Y. Abay and P. A. Kyriacou, "Reflectance photoplethysmography as noninvasive monitoring of tissue blood perfusion," IEEE Trans. Biomed. Eng. 62(9), 2187-2195 (2015).

20. T. Y. Abay and P. A. Kyriacou, "Photoplethysmography for blood volumes and oxygenation changes during intermittent vascular occlusions," J. Clin. Monit. Comput. 32, 447-455 (2018).

21. P. van der Zee et. al., "Experimentally measured optical pathlengths for the adult head, calf and forearm and the head of the newborn infant as a function of inter optode spacing," Adv. Exp. Med. Biol. 316, 143-153 (1992).

22. I. V. Meglinski and S. J. Matcher, "Modelling the sampling volume for skin blood oxygenation measurements," Med. Biol. Eng. Comput. 39(1), 44-50 (2001).

23. D. A. Boas et al., "The accuracy of near infrared spectroscopy and imaging during focal changes in cerebral hemodynamics," Neuroimage 13(1), 76-90 (2001).

24. K. Uludağ et al., "Separability and crosstalk: optimizing dual wavelength combinations for near-infrared spectroscopy of the adult head," Neuroimage 22(2), 583-589 (2004).

25. G. Strangman, M. A. Franceschini, and D. A. Boas, "Factors affecting the accuracy of near-infrared spectroscopy concentration calculations for focal changes in oxygenation parameters," Neuroimage 18(4), 865-879 (2003).

26. A. Jakobsson and G. E. Nilsson, "Prediction of sampling depth and photon pathlength in laser Doppler flowmetry," Med. Biol. Eng. Comput. 31(3), 301-307 (1993).

27. F. F. M. de Mul et al., "Laser Doppler velocimetry and Monte Carlo simulations on models for blood perfusion in tissue," Appl. Opt. 34(28), 6595-6611 (1995).

28. G. B. Hanna et al., "Use of lightguide spectrophotometry to quantify skin oxygenation in a variable model of venous hypertension," $\mathrm{Br}$. $J$. Surg. 82(10), 1352-1356 (1995).

29. L. Wang, S. L. Jacques, and L. Zheng, "MCML-Monte Carlo modeling of light transport in multi-layered tissues," Comput. Meth. Programs Biomed. 47(2), 131-146 (1995).

30. S. A. Prahl, "Light transport in tissue," thesis submitted to University of Texas at Austin (1989).

31. B. C. Wilson and G. Adam, "A Monte Carlo model for the absorption and flux distributions of light in tissue," Med. Phys. 10(1983), 824-830 (1983).
32. S. A. Prahl et al., "Dosimetry of laser radiation in medicine and biology," Proc. SPIE 5, 102-111 (1989).

33. C. Zhu and Q. Liu, "Review of Monte Carlo modeling of light transport in tissues," J. Biomed. Opt. 18(5), 050902 (2013).

34. I. V. Meglinski and S. J. Matcher, "Computer simulation of the skin reflectance spectra," Comput. Meth. Programs Biomed. 70(2), 179-186 (2003).

35. V. V. Tuchin, Advanced Biophotonics: Tissue Optical Sectioning, Taylor \& Francis, CRC Press, Boca Raton (2013).

36. S. Standringet al., "Gray's Anatomy, 39th edition: the anatomical basis of clinical practice," Am. J. Neuroradiol. 26(10), 2703 LP-2704 (2005).

37. A. Doronin, I. Fine, and I. Meglinski, "Assessment of the calibration curve for transmittance pulse-oximetry," Laser Phys. 21(11), 19721977 (2011)

38. A. Doronin and I. Meglinski, "Online object oriented Monte Carlo computational tool for the needs of biomedical optics," Biomed. Opt. Express 2(9), 2461-2469 (2011).

39. V. V. Tuchin et al., "Finger tissue model and blood perfused skin tissue phantom," Proc. SPIE 7898, $78980 Z$ (2011).

40. A. Doronin, "The unified Monte Carlo model of photon migration in scattering tissue-like media for the needs of biomedical optics," thesis submitted to University of Otago (2014).

41. J. M. Schmitt, "Simple photon diffusion analysis of the effects of multiple scattering on pulse oximetry," IEEE Trans. Biomed. Eng. 38(12), 1194-1203 (1991).

42. J. L. Reuss, "Multilayer modeling of reflectance pulse oximetry," IEEE Trans. Biomed. Eng. 52(2), 153-159 (2005).

43. J. L. Reuss and D. Siker, "The pulse in reflectance pulse oximetry: modeling and experimental studies," J. Clin. Monit. Comput. 18(4), 289-299 (2004).

44. J. M. Steinke and A. P. Shepherd, "Diffusion model of the optical absorbance of whole blood," J. Opt. Soc. Am. A. 5(6), 813-22 (1988).

45. N. Bosschaart et al., "A literature review and novel theoretical approach on the optical properties of whole blood," Lasers Med. Sci. 29(2), 453479 (2014).

46. M. Friebel et al., "Determination of optical properties of human blood in the spectral range 250 to $1100 \mathrm{~nm}$ using Monte Carlo simulations with hematocrit-dependent effective scattering phase functions," J. Biomed. Opt. 11(3), 034021 (2006).

47. G. M. Hale and M. R. Querry, "Optical constants of water in the 200-nm to $200-\mu \mathrm{m}$ wavelength region," Appl. Opt. 12(3), 555 (1973).

48. S. L. Jacques and D. J. McAuliffe, "The melanosome: threshold temperature for explosive vaporization and internal absorption coefficient during pulsed laser irradiation," Photochem. Photobiol. 53(6), 769-775 (1991).

49. J. Laufer et al., "Effect of temperature on the optical properties of ex vivo human dermis and subdermis," Phys. Med. Biol. 43, 2479-2489 (1998).

50. A. J. Welch and M. J. C. Van Gemert, Optical-Thermal Response of Laser-Irradiated Tissue, Vol. 2, Springer, New York (2011).

51. J. P. Phillips, P. A. Kyriacou, and D. P. Jones, "Calculation of photon path changes due to scatter in Monte Carlo simulations," Conf. Proc. IEEE Eng. Med. Biol. Soc. 2010, 4959-4962 (2010).

52. S. Chatterjee, J. P. Phillips, and P. A. Kyriacou, "Monte Carlo investigation of the effect of blood volume and oxygen saturation on optical path in reflectance pulse oximetry," Biomed. Phys. Eng. Express 2(6), 65018 (2016)

53. W. G. Zijlstra, A. Buursma, and O. W. van Assendelft, Visible and Near Infrared Absorption Spectra of Human and Animal Haemoglobin Determination and Application, p. 368, VSP BV, The Netherlands (2000).

54. S. Chatterjee, J. P. Phillips, and P. A. Kyriacou, "Investigating optical path in reflectance pulse oximetry using a multilayer Monte Carlo model," Proc. SPIE 10411, 1041106 (2017).

55. D. Y. Churmakov, I. V. Meglinski, and D. A. Greenhalgh, "Influence of refractive index matching on the photon diffuse reflectance," Phys. Med. Biol. 47(23), 4271-4285 (2002).

Subhasri Chatterjee received her BSc degree (2009) in physics and an MSc degree (2011) in biomedical instrumentation from University of Calcutta, India. Afterward, she was awarded with the National Photonics Fellowship of India to work as a research intern in 
Indian Institute of Science Education and Research-Kolkata, India (2011-2013). Currently, she is pursuing her PhD at RCBE, City, University of London under the Erasmus Mundus scholarship program. Her main research interest is biomedical optics.

Tomas Ysehak Abay received his BEng in biomedical engineering from Politecnico di Milano in 2010. After working for one year as a medical engineer at San Matteo Hospital, Pavia, Italy, he later received the MSc in biomedical engineering in 2012 and a $\mathrm{PhD}$ in biomedical engineering in 2016, both from City, University of London. His research focuses on photoplethysmography, pulse oximetry, near-infrared spectroscopy, and other non-invasive optical techniques for monitoring blood volumes and oxygenation.

Justin Paul Phillips received a BSc degree (1992) in physics from Durham University, UK, and a PhD (2009) in biomedical engineering from Queen Mary, University of London, UK. He was awarded a Royal Academy of Engineering Senior Research Fellowship in 2015 and in 2017 he was a visiting faculty researcher at Google. His research covers optical sensors and wearable computing systems for health diagnostics and monitoring.

Panayiotis A. Kyriacou received a BESc degree in electrical engineering from the University of Western Ontario, Canada, and $\mathrm{MSc}$ and $\mathrm{PhD}$ degrees in medical electronics and physics from St. Bartholomew's Medical College, University of London. Currently, he is a professor of biomedical engineering and an associate dean for research and enterprise at the School of Mathematics Computer Science and Engineering at City University London, and the director of the Research Centre for Biomedical Engineering. 